\title{
Publisher Correction: Single-DNA electron spin resonance spectroscopy in aqueous solutions
}

Fazhan Shi (D), Fei Kong (D), Pengju Zhao, Xiaojun Zhang, Ming Chen, Sanyou Chen, Qi Zhang, Mengqi Wang, Xiangyu Ye, Zhecheng Wang, Zhuoyang Qin, Xing Rong, Jihu Su, Pengfei Wang, Peter Z. Qin (iD and Jiangfeng Du (D)

Correction to: Nature Methods https://doi.org/10.1038/s41592-018-0084-1, published online 6 August 2018

In the version of this paper originally published online, the ORCID ID for Peter Z. Qin was incorrectly assigned to Zhuoyang Qin. In addition, the ORCID for Fazhan Shi was omitted. These errors have been corrected in the print, PDF, and HTML versions of the paper.

Published online: 14 August 2018

https://doi.org/10.1038/s41592-018-0119-7

\section{Addendum: Biotinylation by antibody recognition-a method for proximity labeling}

\author{
Daniel Z Bar (iD, Kathleen Atkatsh, Urraca Tavarez, Michael R Erdos D, Yosef Gruenbaum and Francis S Collins (D)
}

Addendum to: Nature Methods 15, 127-133 (2018) https://doi.org/10.1038/nmeth.4533, published online 18 December 2017

In the version of this article initially published, the introduction focused on methods for characterizing the nuclear envelope and did not include a comprehensive overview of proximity-based methods, some of which have similarly utilized antibody-conjugated peroxidase for proximity labeling by biotin deposition ( refs $^{1-4}$ below). The authors believe they should have included these references and apologize for the omission.

\section{References}

1. Kotani, N. et al. Biochemical visualization of cell surface molecular clustering in living cells. Proc. Natl Acad.Sci. USA 105, 7405-7409 (2008).

2. Hashimoto, N. et al. Proteomic analysis of ganglioside-associated membrane molecules: substantial basis for molecular clustering. Proteomics 12, 3154-3163 (2012).

3. Li, X. W. et al. New insights into the DT40 B cell receptor cluster using a proteomic proximity labeling assay. J. Biol. Chem. 289, 14434-14447 (2014).

4. Rees, J. S. et al. Selective proteomic proximity labeling assay using tyramide (SPPLAT): a quantitative method for the proteomic analysis of localized membranebound protein clusters. Curr. Protoc. Protein Sci. 80, 19.27.1-19.27.18 (2015). 